\title{
Immunological detection of fructose-derived advanced glycation end-products
}

\author{
Masayoshi Takeuchi ${ }^{1}$, Mina Iwaki ${ }^{2}$, Jun-ichi Takino ${ }^{1}$, Hikari Shirai ${ }^{1}$, Mihoko Kawakami ${ }^{1}$, Richard Bucala ${ }^{3}$ and \\ Sho-ichi Yamagishi ${ }^{4}$
}

The advanced stage of non-enzymatic glycation (also called the Maillard reaction) that leads to the formation of advanced glycation end-products (AGEs) has an important function in the pathogenesis of angiopathy in diabetic patients. So far, most studies have been focused on the Maillard reaction by glucose. Although an elevated level of glucose had been thought to have a primary function in the Maillard reaction, on a molecular basis, glucose is among the least reactive sugars within biological systems. In addition to the extracellular formation of AGEs, rapid intracellular AGEs formation by various intracellular precursors (fructose, trioses, and dicarbonyl compounds) has recently attached attention. In this study, we considered the Maillard reaction with particular attention to the potential function of fructose. Fructose AGEmodified serum albumins were prepared by incubation of rabbit or bovine serum albumin (RSA or BSA) with D-fructose. After immunization of rabbits, fructose-derived AGEs (Fru-AGE) antiserum was subjected to affinity chromatography on a Sepharose 4B column coupled with Fru-AGE-BSA. Characterization of the novel anti-Fru-AGE antibody was performed with a competitive enzyme-linked immunosorbent assay and immunoblot analysis. The assay of Fru-AGE was established using the immunoaffinity-purified-specific antibody, and the presence of Fru-AGE in healthy and diabetic serum was shown $(7.04 \pm 4.47$ vs $29.13 \pm 18.08 \mathrm{U} / \mathrm{ml})$. We also investigated whether high glucose treatment could stimulate intracellular Fru-AGE production in cultured pericytes, and we analyzed the amount of Fru-AGE contained in some common commercial beverages and condiments. It is possible that Fru-AGE formation by these endogenous and exogenous routes contributes importantly to the tissue pathology of diabetes and aging. This paper provides novel and clinically relevant information on the detection of Fru-AGE between fructose and proteins.

Laboratory Investigation (2010) 90, 1117-1127; doi:10.1038/labinvest.2010.62; published online 8 March 2010

KEYWORDS: advanced glycation end-products (AGEs); diabetes; diet; fructose; fructose-derived AGEs (Fru-AGE); polyol pathway

Fructose is an important dietary source of carbohydrates. Owing to its unique metabolic properties, fructose promotes adverse metabolic changes, including glucose intolerance, hyperlipedemia, hypertension, and hyperuricemia. ${ }^{1-5}$ The two sources for fructose in vivo are the endogenous formation from glucose through the polyol pathway and the exogenous supply from the diet, either as sucrose or free fructose. The polyol pathway, an accessory of glucose metabolism, entails the conversion of glucose to fructose by aldose reductase (AR) and sorbitol dehydrogenase (SDH). ${ }^{6-9}$

So far, most studies have been focused on non-enzymatic glycation (also called the Maillard reaction) by glucose, because glucose is by far the most abundant sugar in blood.
In principle, however, all reducing sugars as well as certain molecules related to sugar, such as ascorbic acid, can initiate the Maillard reaction in vivo. ${ }^{10,11}$ The Maillard reaction is a process in which reducing sugars react spontaneously with the terminal $\alpha$-amino-group or the $\varepsilon$-amino-group of lysine residues in proteins to advanced glycation end products (AGEs). Although an elevated level of glucose had been thought to have a primary function in the Maillard reaction, on a molecular basis, glucose is among the least reactive sugars within biological systems. ${ }^{12}$ In addition to the extracellular formation of AGEs, rapid intracellular AGEs formation by various intracellular precursors (fructose, trioses, and dicarbonyl compounds) has recently attached attention. ${ }^{13,14}$

\footnotetext{
${ }^{1}$ Department of Pathophysiological Science, Faculty of Pharmaceutical Sciences, Hokuriku University, Kanazawa, Japan; ${ }^{2}$ Department of Biochemistry, Faculty of Pharmaceutical Sciences, Hokuriku University, Kanazawa, Japan; ${ }^{3}$ Department of Medicine, Pathology, and Epidemiology and Public Health, Yale University School of Medicine, New Haven, CT, USA and ${ }^{4}$ Department of Pathophysiology and Therapeutics of Diabetic Vascular Complications, Kurume University School of Medicine, Kurume, Japan

Correspondence: Dr M Takeuchi, PhD, Department of Pathophysiological Science, Faculty of Pharmaceutical Sciences, Hokuriku University, Ho-3 Kanagawa-machi, Kanazawa 920-1181, Japan.

E-mail: m-takeuchi@hokuriku-u.ac.jp

Received 26 May 2009; revised 3 January 2010; accepted 18 January 2010
} 
The initial phase of the Maillard reaction with fructose, that is the condensation of the carbonyl group of fructose with amino-groups in proteins, is quite similar to that for glucose (Figure 1). ${ }^{15}$ Although an elevated level of glucose had been thought to have a primary function in the Maillard reaction, the formation of AGEs is now known to result also from the action of various metabolites other than glucose. The realization that fructose and its metabolites, at elevated concentrations, can initiate potentially deleterious changes, such as the non-enzymatic fructosylation of proteins, has raised interest and concern about this property of fructose. ${ }^{16}$

Fructose is considered to contribute to AGEs formation more than glucose because the anomerization equilibrium for fructose is shifted more to the reactive, open chain form of the sugar. ${ }^{12}$ When fructose and protein are incubated in vitro, fluorescent and cross-linking products form, and it has been estimated that fructose produces 10 times more AGEs than does glucose. ${ }^{15,17}$ Although the in vivo formation of fructose-derived AGEs (Fru-AGE) has long been suspected, experimental evidence for their formation has not yet been reported. In this study, we established the antibody against Fru-AGE prepared from rabbit serum albumin (RSA), and investigated the properties of the antibody. We also showed the formation of Fru-AGE in the serum of diabetic patients, culture cells, and food. As the search for relevant diabetes biomarkers is an important one, this study may provide a new index for study in diabetes.

\section{MATERIALS AND METHODS}

\section{Preparation of Various Glycated Proteins}

Fru-AGE-bovine serum albumin (BSA) and Fru-AGE-RSA were prepared as described below. BSA (A0281, SigmaAldrich, St Louis, MO, USA) or RSA (A0764, Sigma-Aldrich) at $25 \mathrm{mg} / \mathrm{ml}$ was incubated under sterile conditions with $0.5 \mathrm{M} \mathrm{D}$-fructose and $5 \mathrm{mM}$ diethylenetriamine-pentaacetic acid (DTPA; Dojindo Laboratories, Kumamoto, Japan) in $0.2 \mathrm{M}$ phosphate buffer ( $\mathrm{pH} 7.4$ ) at $37^{\circ} \mathrm{C}$ for 8 weeks. Lowmolecular weight reactants and fructose were removed using a PD-10 column (GE Healthcare UK, Buckinghamshire, England) chromatography and dialysis against phosphatebuffered saline (PBS). Various AGE-BSAs were prepared as described earlier. ${ }^{18-21}$ Briefly, each protein was incubated under sterile conditions with $0.5 \mathrm{M}$ D-glucose (AGE-1), $0.5 \mathrm{M}$ fructose-1-phosphate (F-1-P-AGE), 0.5 M fructose-6-phosphate (F-6-P-AGE), 0.5 M fructose-1,6-diphosphate (F-1,6DP-AGE), $0.1 \mathrm{M}$ glyceraldehyde (AGE-2), $0.1 \mathrm{M}$ glycolaldehyde (AGE-3), 0.1 M methylglyoxal (AGE-4), $0.1 \mathrm{M}$ glyoxal (AGE-5), or $0.2 \mathrm{M}$ 3-deoxyglucosone (AGE-6) and $5 \mathrm{mM}$ DTPA in $0.2 \mathrm{M}$ phosphate buffer ( $\mathrm{pH} 7.4$ ) at $37^{\circ} \mathrm{C}$ for 7 days, except in the case of incubation with 3-deoxyglucosone (2 weeks) or D-glucose, F-1-P, F-6-P, and F-1,6-DP (8 weeks). Low-molecular weight reactants and sugars or carbonyl compounds were removed using PD-10 column chromatography and dialysis against PBS. Pentosidine-BSA, pyrraline$\mathrm{BSA}$, and argpyrimidine-BSA were obtained from MBC, Tokyo, Japan. $\mathrm{N}$-carboxymethyllysine (CML)-BSA, $\mathrm{N}$-carboxyethyllysine (CEL)-BSA, and $N$-ethyllysine (NEL)-BSA were prepared as described earlier. ${ }^{22}$ Briefly, $50 \mathrm{mg} / \mathrm{ml}$ of BSA was incubated at $37^{\circ} \mathrm{C}$ for $24 \mathrm{~h}$ with $50 \mathrm{mM}$ glyoxylic acid, pyruvic acid, or acetaldehyde and $150 \mathrm{mM}$ sodium cyanoborohydride in $0.2 \mathrm{M}$ phosphate buffer ( $\mathrm{pH} 7.4$ ), followed by PD-10 column chromatography and dialysis against PBS. Fru-AGE was reduced with $\mathrm{NaBH}_{4}$ as described earlier. ${ }^{18}$ Protein concentrations were determined with the Dc protein assay reagent (Bio-Rad Laboratories, Richmond, CA, USA) using BSA as a standard.

\section{Preparation of Anti-Fru-AGE Antiserum}

Four milligrams of Fru-AGE-RSA was emulsified in 50\% Freund's complete adjuvant (Wako Pure Chemical Industries,

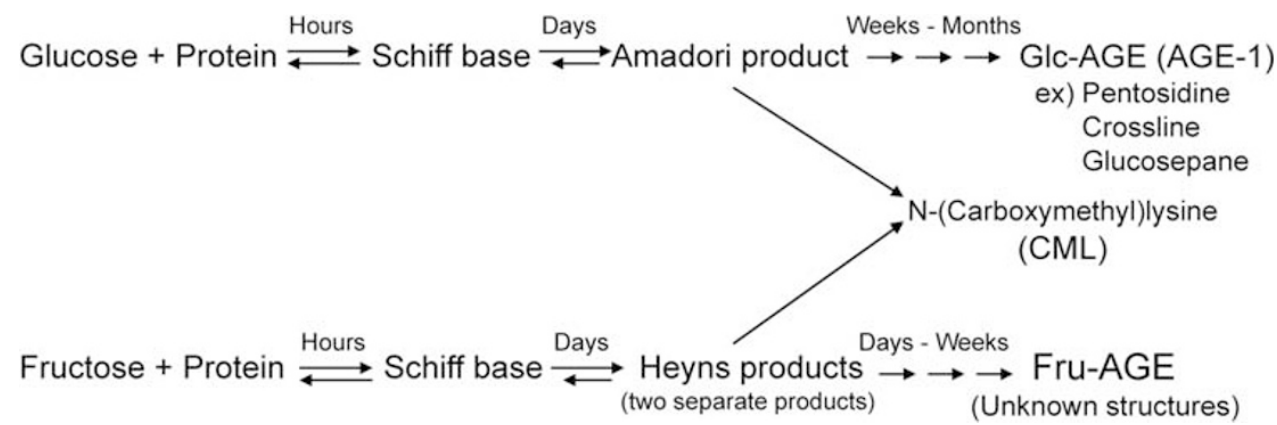

Figure 1 In the pathway for AGEs formation, a non-enzymatic reaction between D-glucose or D-fructose and the N-terminal amino-acid residues and/or $\varepsilon$-amino-groups of a protein initially forms reversible Schiff base adduct. In the case of glucose, the Schiff base adduct then slowly undergoes Amadori rearrangement to produce a more stable, but still slowly reversible adduct. With fructose, the reaction is similar, but the reaction is termed as Heyns products and results in the formation of two products. ${ }^{15}$ These early glycation products undergo further complex reactions such as rearrangement, dehydration, and condensation to become irreversibly cross-linked, heterogeneous fluorescent derivatives termed as advanced glycation end-products (AGEs). $\mathrm{N}$-(carboxymethyl)lysine (CML) can be produced by degradation of the Amadori products. ${ }^{25}$ Still only partially characterized, this end stage of glycation proceeds. Several types of glucose-derived AGEs (Glc-AGE) have recently been structurally characterized, such as CML, pentosidine, crosslines, and glucosepane. $^{35}$ 
Osaka, Japan) and injected intradermally into Japanese white rabbits (Sankyo Labo-service, Tokyo, Japan). This procedure was repeated at weekly intervals for 6 weeks. After a 2-week pause, the rabbits were given a booster injection of $4 \mathrm{mg}$ of the antigen. Animals were bled on the 10th day after this injection and serum was obtained for purification.

\section{Purification of Antibody to Fru-AGE from Anti-Fru-AGE Antiserum by Affinity Chromatography}

Antibody specific for Fru-AGE was isolated from the abovementioned rabbit antiserum by affinity chromatography. Fru-AGE-BSA or CML-BSA ( $125 \mathrm{mg}$ of each protein) was coupled to $25 \mathrm{ml}$ of $\mathrm{CNBr}$-activated Sepharose $4 \mathrm{~B}$ (GE Healthcare UK) according to the manufacturer's instructions. Then $25 \mathrm{ml}$ of rabbit antiserum was applied to a column $(2.5 \times 5.5 \mathrm{~cm})$ of Sepharose 4B coupled with Fru-AGE-BSA. After extensive washing with PBS, the adsorbed fractions were eluted with $20 \mathrm{mM}$ sodium phosphate buffer containing $1 \mathrm{M}$ potassium thiocyanate $(\mathrm{pH}$ 7.4). The anti-Fru-AGE antibody fractions were pooled, concentrated using Centriprep-10 (Millipore Corporation, Billeria, MA, USA), and passed through a PD-10 column equilibrated with PBS. The anti-Fru-AGE antibody thus obtained then was loaded onto a column $(1.5 \times 5.5 \mathrm{~cm})$ of Sepharose $4 \mathrm{~B}$ coupled with CMLBSA, which was washed with $30 \mathrm{ml}$ of PBS to obtain the unadsorbed fraction (Fru-AGE antibody). The adsorbed fraction (CML antibody) was eluted with $20 \mathrm{ml}$ of $20 \mathrm{mM}$ sodium phosphate buffer containing $1 \mathrm{M}$ potassium thiocyanate $(\mathrm{pH} 7.4)$. Fractions $(1.0 \mathrm{ml})$ were monitored for absorbance at $280 \mathrm{~nm}$. The unadsorbed fractions were pooled, concentrated with Centriprep-10, and passed through a PD-10 column equilibrated with PBS for use in this study.

\section{Competitive Enzyme-Linked Immunosorbent Assay}

Ligand inhibition and Fru-AGE measurements were performed with a competitive enzyme-linked immunosorbent assay (ELISA) by using immunoafinity-purified anti-FruAGE antibody, as described earlier. ${ }^{18,19,21}$. Briefly, 96-well EIA/ RIA plate (flat bottom without lid, high binding; Corning Incorporated, Corning, NY, USA) was coated with $1 \mu \mathrm{g} / \mathrm{ml}$ Fru-AGE to each well and incubated for overnight at $4{ }^{\circ} \mathrm{C}$. Wells were washed three times with $0.3 \mathrm{ml}$ of washing solution (PBS containing 0.05\% Tween-20). Wells were then blocked by incubation for $1 \mathrm{~h}$ with $0.2 \mathrm{ml}$ of a solution of PBS containing 1\% BSA. After washing with washing solution, test samples $(50 \mu \mathrm{l})$ were added to each well as a competitor for $50 \mu \mathrm{l}$ of immunoaffinity-purified anti-Fru-AGE antibody (1:2500), followed by incubation for $2 \mathrm{~h}$ at room temperature with gentle shaking on a horizontal rotary shaker. Then, wells were washed with washing solution and developed with an alkaline phosphatase-conjugated sheep anti-rabbit IgG (Millipore Corporation) using p-nitrophenyl phosphate as the colorimetric substrate (Pierce, Rockford, IL, USA). The results for specificity were expressed as $B / B_{0}$, which was calculated as follows: (experimental OD - background OD)/ (total OD - background OD).

\section{Measurement of Fru-AGE Content}

Samples of sera and beverages were measured with competitive ELISA after they were diluted $>20$-fold. ELISA values of serum AGEs remained constant irrespective of whether serum was diluted 10- or 100-fold (after controlling for dilution). The Fru-AGE content of each sample was read from the calibration curve for Fru-AGE-BSA and was expressed as Fru-AGE units (U) per ml, where $1 \mathrm{U}$ corresponds to $1 \mu \mathrm{g}$ of Fru-AGE-BSA standard.

\section{Determinations of Clinical Parameters}

HbAlc was analyzed with a high-performance liquid chromatography method (HA8131, Kyoto Daiichi Kagaku, Kyoto, Japan) that has a normal range of $4.3-5.8 \%$ with an interassay coefficient of variation of $5-8 \%$. Blood glucose, triglycerides, and total cholesterol were measured enzymatically.

\section{Immunoblot Analysis}

AGE proteins were subjected to electrophoresis on $7.5 \%$ sodium dodecyl sulfate (SDS) polyacrylamide gel (PAGE) (Atto, Tokyo, Japan). The proteins then were transferred electrophoretically to polyvinyliden difuoride membranes (Atto) for $30 \mathrm{~min}$ at $2.5 \mathrm{~mA}$ per $\mathrm{cm}^{2}$. Each membrane was blocked with $4 \%$ skim milk in PBS for $1 \mathrm{~h}$ at room temperature, reacted for $2 \mathrm{~h}$ with immunoaffinity-purified anti-Fru-AGE antibody (1:2500), washed three times for $5 \mathrm{~min}$ each with washing solution, incubated for $1 \mathrm{~h}$ in $4 \%$ skim milk in PBS with 1:2000 diluted alkaline phosphataseconjugated sheep anti-rabbit IgG, washed five times for $5 \mathrm{~min}$ each with washing solution, and finally incubated with 5-bromo-4-chloro-3-indoyl phosphate-nitroblue tetrazolium (Wako Pure Chemical Industries).

\section{Size Distribution of Fru-AGE in Sera from Normal Controls and Diabetic Patients on Hemodialysis}

Ten milliliters of sera from each of three normal individuals and from three diabetic patients with end-stage renal disease on hemodialysis were concentrated by lyophilization and dissolved in $2 \mathrm{ml}$ of distilled water. These solutions were applied to a Sephacryl S-200 column $(1.5 \times 110 \mathrm{~cm})$, which was equilibrated with PBS ( $\mathrm{pH} 7.4)$ and eluted with the same buffer (fraction size: $1.5 \mathrm{ml}$, flow rate: $10 \mathrm{ml} / \mathrm{h}$ ) in a cold room. The molecular weight standards used were aldolase (160 kDa), BSA (67 kDa), chymotrypsinogen A (25 kDa), and vitamin $\mathrm{B}_{12}(1.3 \mathrm{kDa})$. Each fraction was monitored for absorbance at $280 \mathrm{~nm}$ and the Fru-AGE activity of each fraction was measured by Fru-AGE-ELISA and characteristic AGEs-specific fluorescence $(\mathrm{Ex}=360 \mathrm{~nm} / \mathrm{Em}=420 \mathrm{~nm})$. 


\section{Cell Culture}

Pericytes were isolated from bovine retina and maintained in Dulbecco's-modified Eagle's medium (Gibco BRL, Rockville, MD, USA) supplemented with $20 \%$ of fetal bovine serum (FBS) (ICN Biomedicals, Aurora, OH, USA). High glucose treatments were carried out in a medium containing $2 \%$ FBS. Medium was changed every 2 days.

\section{Transfection of Human SDH cDNA into Pericytes}

A cDNA coding for human SDH was amplified by polymerase chain reaction (PCR) using a human microvascular endothelial cell lambda cDNA library (Stratagene, La Jolla, CA, USA) as a template and two oligonucleotide primers (5'-GCACTCCAGAGCCAAAAGAG- $3^{\prime}$ and $5^{\prime}$-CTGAGATCC CAAGACTGTGG- $\left.3^{\prime}\right){ }^{23}$ The PCR-amplified cDNA fragment was then cloned into an expression vector, pBK-CMV (Stratagene). Pericytes were transiently transfected with either human SDH cDNA or an empty vector using FuGENE 6 transfection reagent (Roche Diagnostics, Mannheim, Germany) according to the manufacture's instructions. The expression of SDH gene in pericytes was verified by reverse transcription-PCR (RT-PCR).

Measurement of Intracellular Fructose Level in Pericytes SDH-transfected or non-transfected pericytes were treated with 5 or $30 \mathrm{mM}$ glucose in the presence or absence of $0.3 \mu \mathrm{M}$ fidarestat, a potent AR inhibitor, for 6 days. Then intracellular fructose level was determined by a gas chromatography with tandem mass spectrometry (GC/MS), according to the method of Guerrant and Moss ${ }^{24}$

\section{Statistical Analysis}

Some values were presented as means \pm s.e.m. Statistical significance was evaluated using Student's $t$-test for paired comparison; $P<0.05$ was considered significant.

\section{RESULTS}

\section{Characterization of the Immunogen}

The formation of AGEs by RSA (Fru-AGE-RSA) during incubation with D-fructose for 8 weeks was monitored by AGEs-specific fluorescence. Fluorescence was only detectable when the Fru-AGE-RSA complex became light yellowish brown, and was not observed in unglycated RSA after 8 weeks of incubation without D-fructose (Figure 2a). The emission maximum of Fru-AGE-RSA was $420 \mathrm{~nm}$, which is in close agreement with the values for other AGE-RSAs. ${ }^{18,19,21}$ SDSPAGE under reducing conditions revealed that unglycated RSA migrated at $67 \mathrm{kDa}$, whereas Fru-AGE-RSA migrated more slowly than unglycated RSA (Figure 2b), suggesting that covalently linked end products of RSA had been formed non-enzymatically during incubation.

\section{Characterization of Anti-Fru-AGE Antiserum}

We prepared anti-Fru-AGE antiserum in rabbits immunized with Fru-AGE-RSA. This antiserum was applied to an affinity
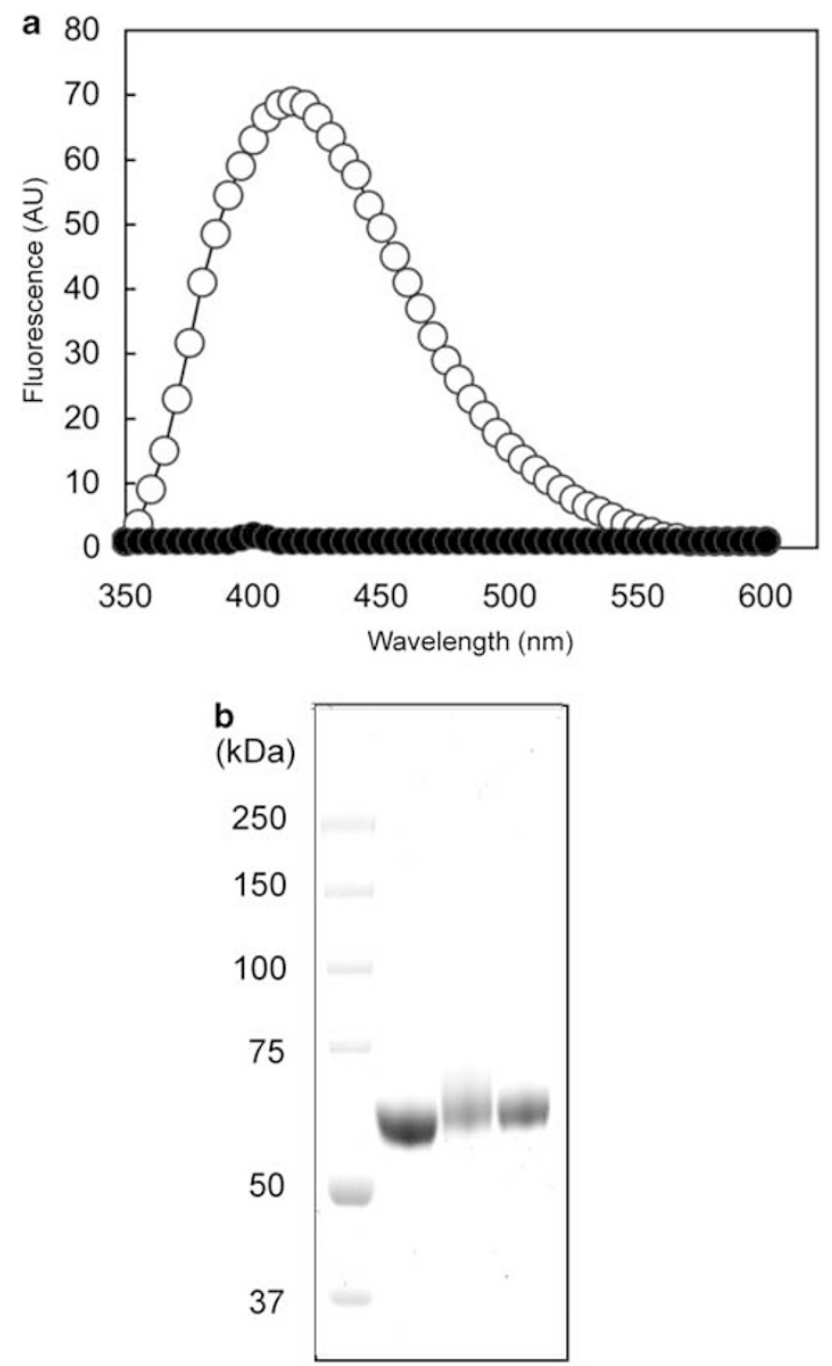

(1) (2) (3)

Figure 2 Characteristics of rabbit serum albumin incubated with D-fructose (Fru-AGE-RSA). Rabbit serum albumin $(25 \mathrm{mg} / \mathrm{ml})$ was incubated under sterile conditions with $0.5 \mathrm{M} \mathrm{D}$-fructose (more than purity $99.5 \%$ ) and $5 \mathrm{mM}$ DTPA in $0.2 \mathrm{M}$ phosphate buffer $(\mathrm{pH} 7.4)$ at $37^{\circ} \mathrm{C}$ for 8 weeks and then low-molecular weight reactants and D-fructose were removed using PD-10 column and dialysis against phosphate-buffered saline (PBS, pH 7.4). (a) Fluorescence emission spectra of $1.0 \mathrm{mg} / \mathrm{ml}$ Fru-AGE-RSA $(O)$ and unglycated RSA (-) at $360 \mathrm{~nm}$. AU: arbitrary units. (b) SDS-PAGE of Fru-AGE-RSA. A total of $2.5 \mu \mathrm{g}$ of unglycated RSA (without D-fructose, lane 1), AGE-1-RSA (incubation with D-glucose, lane 2), and Fru-AGE-RSA (incubation with D-fructose, lane 3) were loaded onto a 7.5\% SDSpolyacrylamide gel. Staining of the gel was performed with Coomassie Brilliant Blue. Size markers $(\mathrm{kDa})$ are shown on the left.

column coupled with Fru-AGE-BSA to obtain a purified anti-Fru-AGE antibody. Figure 3 shows the reactivity for FruAGE-BSA, CML-BSA, and unglycated BSA of the antiserum obtained from rabbit immunized with Fru-AGE-RSA. The antiserum reacted with Fru-AGE-BSA, whereas no reaction was observed with unglycated BSA that had been incubated without D-fructose (Figure 3a). In cross-reactivity studies, 

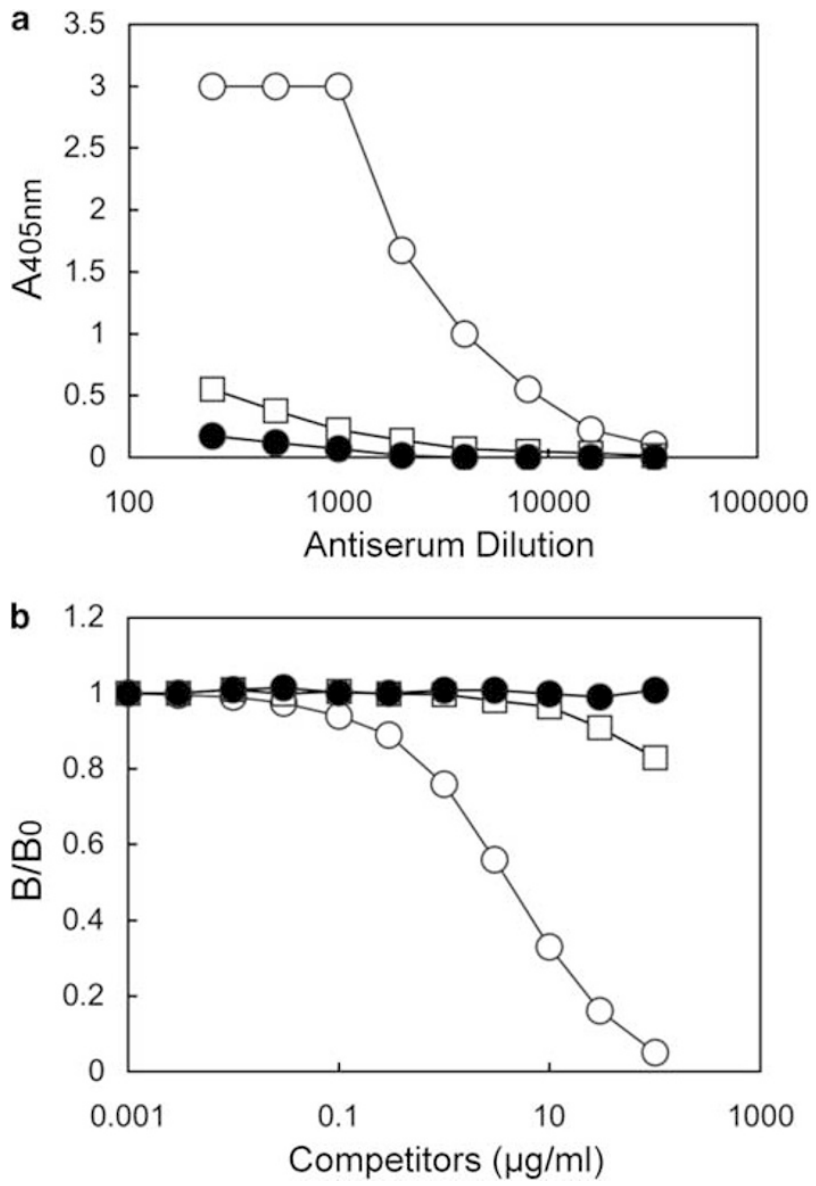

Figure 3 Immunoreactivity of anti-Fru-AGE antiserum. The immunoreactivity of anti-Fru-AGE antiserum with Fru-AGE-BSA $(O)$, CML-BSA $(\square)$, and unglycated BSA $(\bullet)$ was determined at various concentrations by a competitive ELISA. Details are described in MATERIALS AND METHODS. (a) Non-competitive ELISA using bound ligand.

(b) Competitive ELISA using bound ligand, and Fru-AGE-BSA, CML-BSA, or unglycated BSA as competitors.

this antibody also showed a weak reaction with CML-BSA. It, therefore, seemed likely that the antiserum not only contained an antibody specific for Fru-AGE, but also an antibody for CML (Figure 3b). CML can be produced by degradation of the Amadori products or by direct reaction of lysine with some dicarbonyl compounds derived from sugar oxidation. ${ }^{25}$

\section{Separation of Anti-Fru-AGE Antibody from Anti-Fru-AGE Antiserum}

The anti-Fru-AGE antiserum was purified by Fru-AGE-BSA affinity chromatography, and then further separated by CML-BSA affinity chromatography (Figure 4 ). We calculated the percentage of antibodies bound to the CML affinity gel (eluted as the second peak) relative to that of antibody unbound to the affinity gel (eluted as the first peak). Unbound anti-Fru-AGE antibody accounted for $85 \%$ of all antibodies obtained.

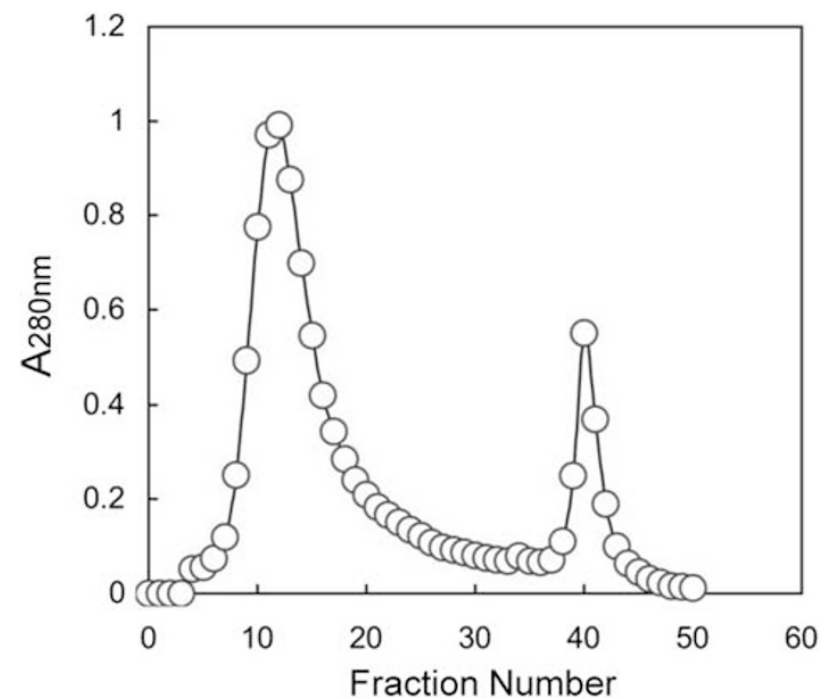

Figure 4 Separation of anti-Fru-AGE antibody from anti-Fru-AGE antiserum by CML-BSA affinity chromatography. Affinity chromatography was performed with polyclonal anti-Fru-AGE antiserum. Details are described in MATERIALS AND METHODS.

\section{Characterization of Anti-Fru-AGE Antibody}

Anti-Fru-AGE antibody obtained by CML-BSA affinity chromatography was used in a competitive ELISA with a glycated human serum albumin (HSA) and various AGE proteins. First, to determine whether Amadori products were recognized by immunoaffinity-purified anti-Fru-AGE antibody, Fru-AGE was reduced with $\mathrm{NaBH}_{4}$. After reduction, the immunoreactivity of Fru-AGE remained essentially unchanged (data not shown). Furthermore, anti-Fru-AGE antibody binding to Fru-AGE was not altered by the addition of glycated HSA (3 mol hexose (as fructosamine) per mole of albumin; Sigma-Aldrich) (data not shown), indicating that early glycation adducts/products as Schiff base and Amadori produces were not prominent immunoreactive epitopes. We next investigated whether immunoaffinity-purified anti-FruAGE antibody could react with fructose-1-phosphate-derived AGEs (F-1-P-AGE), fructose-6-phosphate-derived AGEs (F-6-P-AGE), and fructose-1,6-diphosphate-derived AGEs (F-1,6-DP-AGE). These compounds did not inhibit the binding of immunoaffinity-purified anti-Fru-AGE antibody to Fru-AGE-BSA (Figure 5a). To determine which AGEs were recognized by the immunoaffinity-purified anti-Fru-AGE antibody, BSA was incubated with D-glucose (AGE-1), glyceraldehyde (AGE-2), glycolaldehyde (AGE-3), methylglyoxal (AGE-4), glyoxal (AGE-5), and 3-deoxyglucosone (AGE-6), and the products tested for reactivity in a competitive ELISA (Figure 5b). Furthermore, to examine whether earlier characterized AGEs were recognized by this antibody, pentosidine-BSA, pyrraline-BSA, argpyrimidine-BSA, CMLBSA, CEL-BSA, and NEL-BSA were tested and found not to inhibit the binding of immunoaffinity-purified antiFru-AGE antibody to Fru-AGE-BSA, even when added 

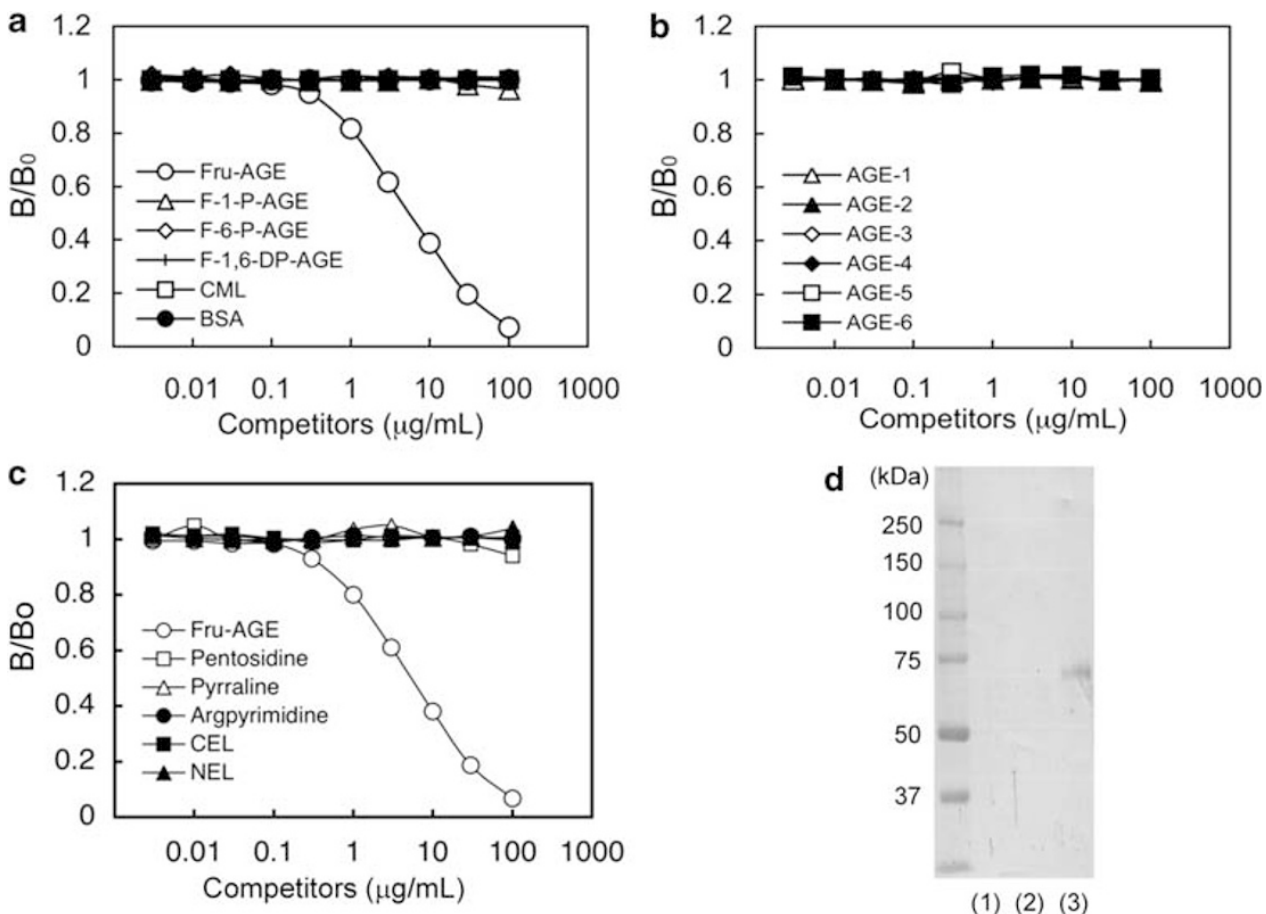

(1) (2) (3)

Figure 5 Immunoreactivity of immunoaffinity-purified anti-Fru-AGE antibody with various AGE proteins. (a-c) Characterization of the immunoaffinitypurified anti-Fru-AGE antibody obtained by CML-BSA affinity chromatography was performed using a competitive ELISA with several AGE-modified proteins (Fru-AGE, fructose-derived AGEs; F-1-P-AGE, fructose-1-phosphate-derived AGEs; F-6-P-AGE, fructose-6-phosphate-derived AGEs; F-1,6-DP-AGE, fructose-1,6diphosphate-derived AGEs; AGE-1, glucose-derived AGEs; AGE-2, glyceraldehyde-derived AGEs; AGE-3, glycolaldehyde-derived AGEs; AGE-4, methylglyoxalderived AGEs; AGE-5, glyoxal-derived AGEs; AGE-6, 3-deoxyglucosone-derived AGEs, $\mathrm{N}$-carboxymethylysine; CML, $\mathrm{N}$-carboxyethyllysine; $\mathrm{CEL}, \mathrm{N}$-ethyllysine; NEL). Pentosidine, pyrraline, argpyrimidine, CML, CEL, and NEL were well-characterized AGE structures. (d) Immunoblot analysis of immunoaffinity-purified anti-Fru-AGE antibody. Aliquots $(0.25 \mu \mathrm{g})$ of unglycated BSA (without D-fructose, lane 1), AGE-1-BSA (incubation with D-glucose, lane 2), and Fru-AGE-BSA (incubation with D-fructose, lane 3) were loaded onto a 7.5\% SDS-polyacrylamide gel. Formation of Fru-AGE in the protein was determined by immunoblot analysis using immunoaffinity-purified anti-Fru-AGE antibody. Size markers $(\mathrm{kDa})$ are shown on the left.

in high concentrations (Figure $5 \mathrm{a}$ and $\mathrm{c}$ ). As shown in Figure $5 \mathrm{a}-\mathrm{c}$, only Fru-AGE specifically inhibited the binding of immunoaffinity-purified anti-Fru-AGE antibody. Immunoblot analysis with immunoaffinity-purified antiFru-AGE antibody confirmed the results obtained by ELISA (Figure 5d). These findings, taken together, indicate that Fru-AGE-BSA had the highest reactivity for immunoaffinitypurified anti-Fru-AGE antibody of the different products tested.

\section{Detection of Serum Fru-AGE}

The amount of Fru-AGE in a healthy person and a type 1 diabetic serum (12 samples of each subjects) was measured by competitive ELISA using immunoaffinity-purified anti-Fru-AGE antibody. As shown in Figure 6, the amount of Fru-AGE was four-fold higher in diabetic than in nondiabetic serum $(29.13 \pm 18.08$ vs $7.04 \pm 4.47 \mathrm{U} / \mathrm{ml})$. However, serum levels of Fru-AGE were not correlated to HbAlc, fasting blood glucose (FBG), triglycerides, and total cholesterol levels in the type 1 diabetic patients (data not shown)

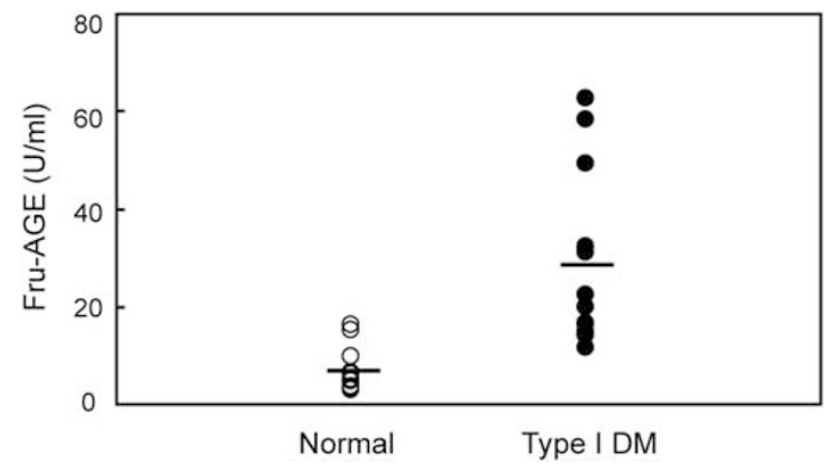

Figure 6 Fru-AGE levels in serum from healthy control (normal) and type 1 diabetic patients (DM). This study involved 12 of each healthy volunteers $(\mathrm{HbA} 1 \mathrm{c}=4.7 \pm 0.1 \%)$ and patients with type 1 diabetes $(\mathrm{HbA} 1 \mathrm{c}=8.5 \pm 0.1 \%)$ without renal dysfunction. Serum levels of Fru-AGE were measured with a competitive ELISA using immunoaffinity-purified anti-Fru-AGE antibody, as described in MATERIALS AND METHODS. $1 \mathrm{U}$ corresponds to $1 \mu \mathrm{g}$ of Fru-AGE-BSA standard.

\section{Size Distribution of Fru-AGE in Sera from Normal Controls and from Diabetic Patients on Hemodialysis}

Sera from both normal controls and diabetic patients with end-stage renal disease on hemodialysis (DM-HD) was 
subjected to Sephacryl S-200 column chromatography and the elution profile of the proteins and peptides monitored by absorbance at $280 \mathrm{~nm}$, AGEs-specific fluorescence, and reactivity with anti-Fru-AGE-specific antibody (Figure 7). Fru-AGE eluted as major peak with an apparent molecular
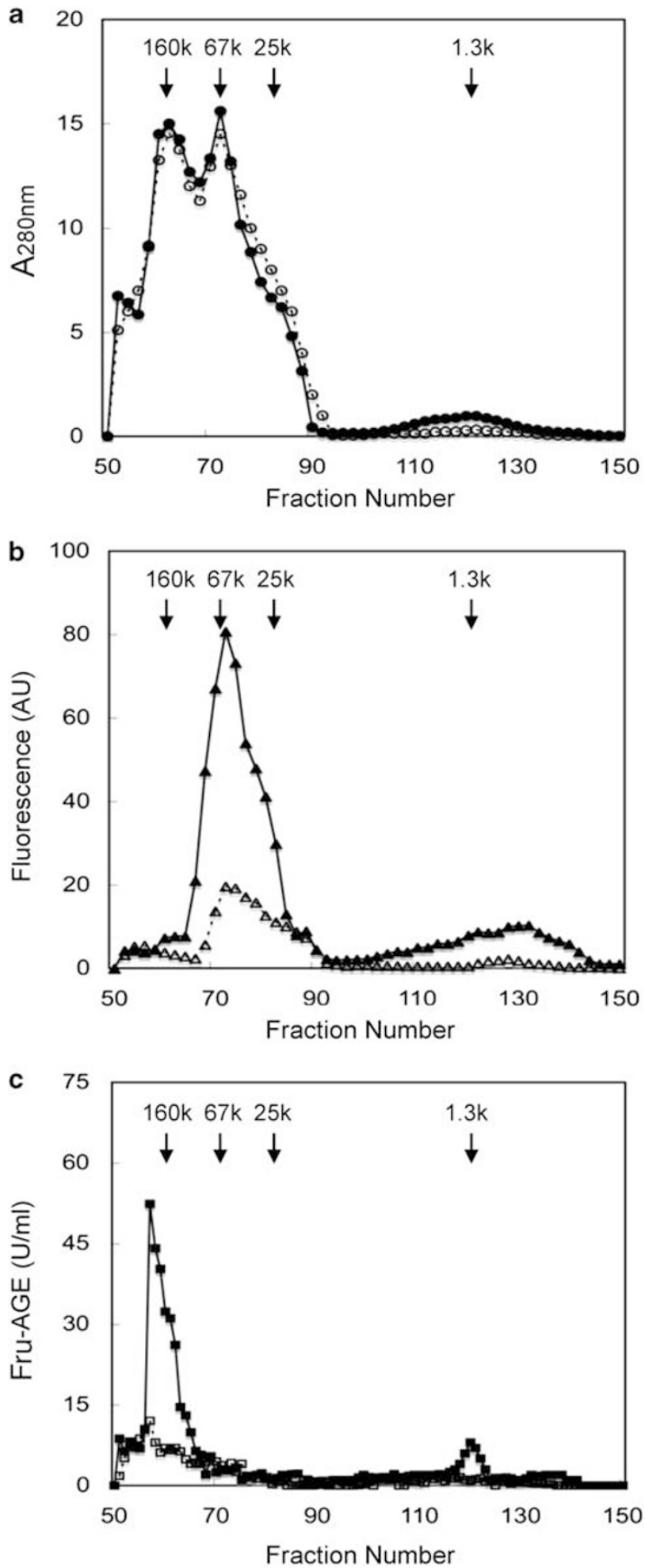

weight of $200 \mathrm{kDa}$. Our earlier papers showed that AGE-1 immunoreactive material was eluted as two peaks with an apparent molecular weight of 200 and $65 \mathrm{kDa} .{ }^{18}$

\section{Detection of Intracellular Fru-AGE}

There is a growing body of evidence that acceleration of the polyol pathway is implicated in the pathogenesis of diabetic vascular complications. ${ }^{26}$ Although Fru-AGE may be formed intracellularly as a consequence of the polyol pathway, this possibility remains unstudied. Accordingly, we examined cultured bovine retinal capillary pericytes as a model system to investigate the formation of intracellularly Fru-AGE.

The amounts of fructose and Fru-AGE in cultured pericytes were measured using GC/MS and competitive ELISA, respectively. Fructose levels were measured under four conditions. No significant difference in levels of fructose was observed after raising the glucose concentration from $5 \mathrm{mM}$ (control) to $30 \mathrm{mM}$ (HG). However, in $\mathrm{HG}+\mathrm{SDH}$ (SDH over-expression), the level of fructose increased about 10 -fold (Figure 8a). We then investigated whether high glucose treatment could stimulate intracellular Fru-AGE production in cultured pericytes. When compared with control, HG alone did not increase Fru-AGE production in cultured pericytes; however, $\mathrm{SDH}$ over-expression was found to significantly stimulate Fru-AGE generation in HG-treated pericytes (Figure $8 \mathrm{~b}$ ). Fidarestat, a potent AR inhibitor, was found to completely prevent fructose and Fru-AGE accumulation in pericytes exposed to HG (Figure 8). The amount of intracellular Fru-AGE was greatly influenced by activation of the polyol pathway, especially fructose concentration level.

\section{Detection of Exogenous Fru-AGE}

Recently, AGEs have been shown to have a function in the development of diabetes by inducing islet $\beta$-cell damage and/ or insulin resistance. ${ }^{27,28}$ In addition, diet is a major environmental source of pro-inflammatory AGEs, and digested food-derived AGEs may have an important function in the development of AGE-related disorders. ${ }^{29,30}$ High levels of fructose are present in certain beverages and condiments. ${ }^{31}$

Figure 7 Size distribution of Fru-AGE in serum from normal control and from diabetic patients on hemodialysis. Sera from normal control or from diabetic patients with end-stage renal disease on hemodialysis (DM-HD) were subjected to gel filtration chromatography (Sephacryl S-200 column: $1.5 \times 110 \mathrm{~cm})$. The eluted fractions were monitored for absorbance at $280 \mathrm{~nm}(\mathrm{O}$, normal control serum; $\bullet, \mathrm{DM}-\mathrm{HD}$ serum), AGE fluorescence $(\Delta$, normal control serum; $\boldsymbol{\Delta}$, DM-HD serum), and Fru-AGE by ELISA ( $\square$, normal control serum; $\mathbf{\square}, \mathrm{DM}-\mathrm{HD}$ serum). The elution of aldolase (160 kDa), BSA (67 kDa), chymotrypsinogen A (25 kDa), and vitamin $B_{12}(1.3 \mathrm{kDa})$ (molecular weight standards) is indicated by arrows. (a and $\mathbf{b})$ Chromatography monitored by absorbance at $280 \mathrm{~nm}$ (a) and AGE fluorescence $(E x=360 \mathrm{~nm} / E m=420 \mathrm{~nm}$ ) (b). (c) Fru-AGE level of each fractions was determined by competitive ELISA using immunoaffinitypurified anti-Fru-AGE antibody, as described in MATERIALS AND METHODS. $1 \mathrm{U}$ corresponds to $1 \mu \mathrm{g}$ of Fru-AGE-BSA standard. 

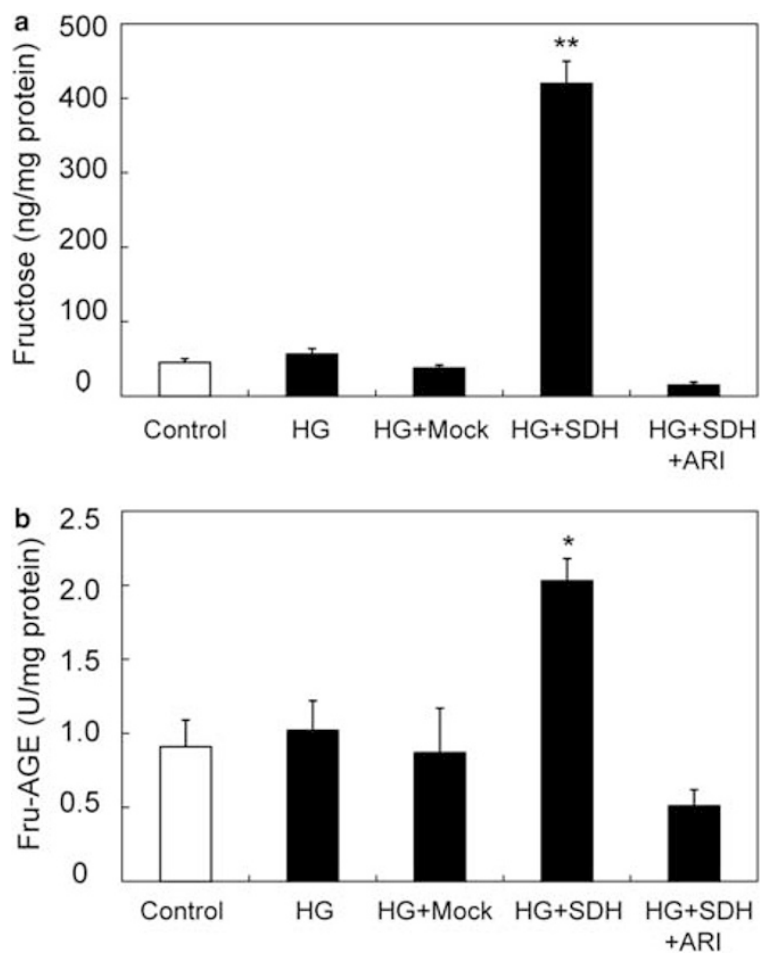

Figure 8 Fructose and Fru-AGE levels in cultured pericytes. Effects of $5 \mathrm{mM}$ glucose (Control) or $30 \mathrm{mM}$ glucose $(\mathrm{HG})$ on intracellular fructose and Fru-AGE levels in cultured pericytes. SDH-transfected or non-transfected pericytes were treated with 5 or $30 \mathrm{mM}$ glucose in the presence or absence of $0.3 \mu \mathrm{M}$ fidarestat for 6 days. (a) Intracellular fructose level was determined by GC/MS. (b) Intracellular Fru-AGE level was determined by competitive ELISA using immunoaffinity-purified anti-Fru-AGE antibody, as described in MATERIALS AND METHODS. $1 \mathrm{U}$ corresponds to $1 \mu \mathrm{g}$ of Fru-AGE-BSA standard as described earlier. ${ }^{*}$ and ${ }^{* *}$ are $P<0.05$ and $P<0.01$ compared with fructose and Fru-AGE levels of the control cells, respectively.
Accordingly, we considered that Fru-AGE formed in the body or provided by the diet was recognized by the prepared antiFru-AGE antibody in this experiment. The Fru-AGE content was measured in $\sim 100$ different commercially available products. The values for Fru-AGE in typical products are shown in Figure 9 by each classification. The highest levels of Fru-AGE were observed in some kind of lactic acid bacteriabased beverages. These beverages contain large quantities of skim milk and simple sugars are included in the raw material. It is possible that the Maillard reaction product forms readily during the production process for these beverages.

\section{DISCUSSION}

The Maillard reaction is a process in which reducing sugars react spontaneously with amino-groups in proteins to AGEs. Although an elevated level of glucose has been considered to have a primary function in the Maillard reaction, on a molecular basis, glucose is among the least reactive sugars within biological systems. ${ }^{12,32}$ The formation of AGEs is now known to result from the action of various metabolites other than glucose, which are primarily located intracellularly and participate in the non-enzymatic glycation reaction at a much faster rate, such as fructose, trioses, and dicarbonyl compounds. ${ }^{33-35}$ In this study, we considered the glycation reaction with particular attention to the potential function of fructose. First, to detect Fru-AGE, an anti-Fru-AGE-specific antibody was produced (Figures 2-4) and the assay of Fru-AGE by a competitive ELISA was established. The immunoaffinity-purified anti-Fru-AGE antibody recognized only a Fru-AGE structure that displayed fluorescence and was not reducible by $\mathrm{NaBH}_{4}$, suggesting the formation of specific

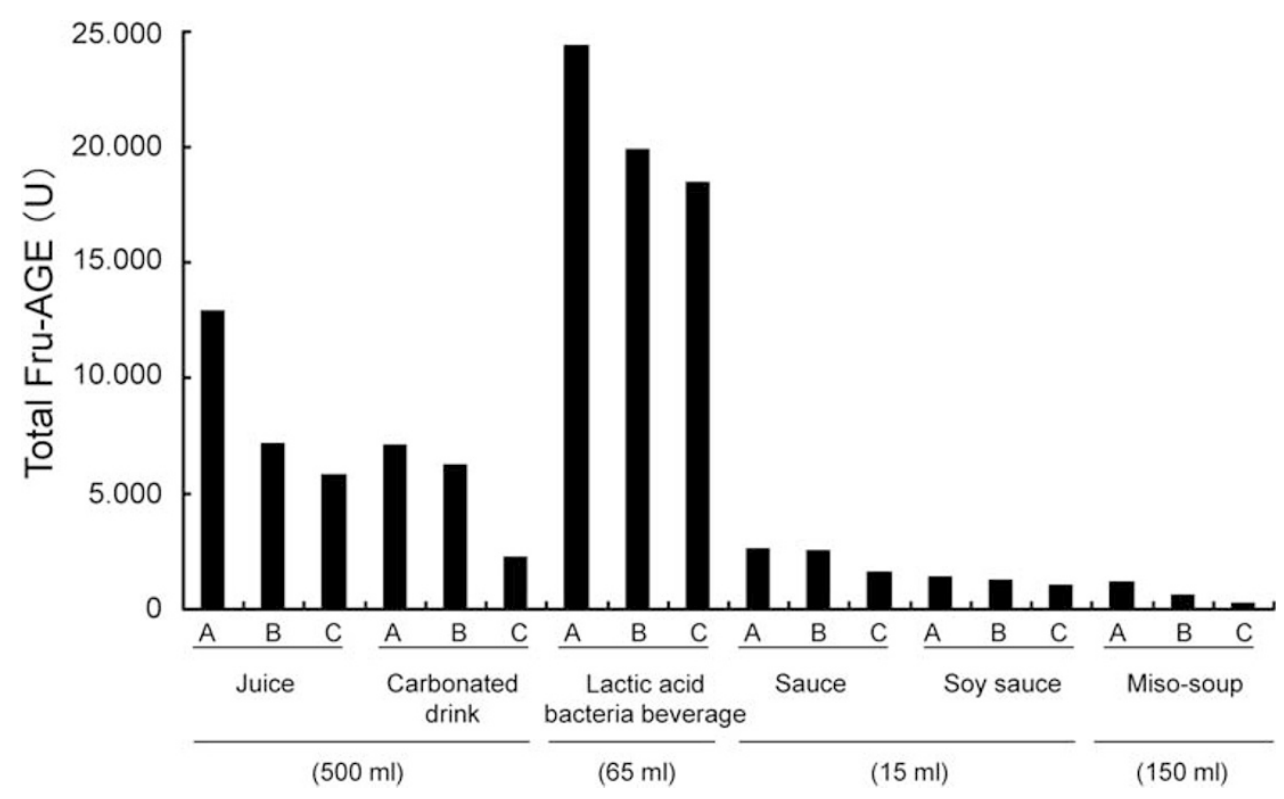

Figure 9 Fru-AGE levels in common beverages and condiments. Fru-AGE level was measured with a competitive ELISA using immunoaffinity-purified antiFru-AGE antibody, as described in MATERIALS AND METHODS. $1 \mathrm{U}$ corresponds to $1 \mu \mathrm{g}$ of Fru-AGE-BSA standard. Respectively, the amount of Fru-AGE of upper 3 products of each classification was shown. A trade name is kept secret (indicated with A, B, or C). 
AGE structures by incubation of the protein with fructose. The epitope of the Fru-AGE seems to be different from earlier characterized AGE structures such as pentosidine, pyrraline, argpyrimidine, CML, CEL, or NEL, or other reducing sugarderived and dicarbonyl-derived AGEs (Figure $5 \mathrm{a}-\mathrm{c}$ ), because BSA preparations conjugated with these compounds were not recognized by immunoaffinity-purified anti-Fru-AGE antibody. Nevertheless, we do not know at present the precise structure of this Fru-AGE, which must await spectroscopic and biochemical analysis.

Next, Fru-AGE of the serum was examined. Fru-AGE was present in the serum of normal individuals and in markedly increased levels in serum from diabetic patients (Figure 6). However, Fru-AGE level in diabetic patients was not correlated with FBG and $\mathrm{HbAlc}$. In our earlier paper, ${ }^{18}$ the serum AGE-1 level in diabetic patients was a weak but significantly correlated with FBG level over the earlier 2 months $(r=0.498, \quad P<0.0001)$ and with HbAlc $(r=0.375$, $P=0.0019)$, but the CML level was not correlated with these clinical parameters. Our earlier papers showed that AGE-1 and CML immunoreactive material was eluted as two peaks with an apparent molecular weight of 200 and $65 \mathrm{kDa}$, whereas another AGEs (AGEs-2 to -6) immunoreactive material was eluted as one peak with an apparent molecular weight of $200 \mathrm{kDa} .^{18,19,21}$ The estimated weight of the AGE proteins detected by our AGEs-ELISA using a specific antiFru-AGE antibody was $200 \mathrm{kDa}$ (Figure 7). Globulins and lipoproteins are presumed as candidate proteins as their molecular weight is $\sim 200 \mathrm{kDa}$.

We suggest that fructose contributes importantly to AGEs formation because of its approximately eightfold higher reactivity. ${ }^{36}$ Intracellularly, fructose is elevated in a number of tissues of diabetic patients in which the polyol pathway is active. In the cells of these tissues, the concentrations of fructose and glucose are of the same magnitude. ${ }^{36}$ Although direct evidence is not yet available, it is likely that the high reactivity of fructose may contribute substantially to the formation of intracellular Fru-AGE and may lead to alteration in the function of cellular proteins, the dysfunction of cells and, subsequently, to vascular complications. To establish the presence of intracellular Fru-AGE, fructose and Fru-AGE in cultured pericytes as a model system were examined. Both the intracellular fructose concentration and the amount of Fru-AGE increased after SDH over-expression under high glucose exposure (Figure 8). Fidarestat, a potent AR inhibitor, was found to completely prevent fructose and Fru-AGE accumulation in pericytes exposed to HG (Figure 8). The amount of intracellular Fru-AGE was greatly influenced by activation of the polyol pathway, especially fructose concentration level. The formation of Fru-AGE in vivo is dependent on the turnover rate of the chemically modified target, the time available, and the fructose concentration.

In the world, most people consume a combination of the two simple sugars, either as sucrose (table sugar) or high- fructose corn syrup (HFCS), used in many sweetened beverages and commercial products. Fructose is one of the most commonly ingested carbohydrates ${ }^{37}$ and dietary fructose intake has increased markedly over the past 30 years. ${ }^{38}$ It is possible that Fru-AGE is formed as a consequence of everyday intake of such diets. It has been reported that the amount of CML and fluorescence intensity in blood is increased in vegetarians with a high-fructose intake. ${ }^{39}$ Our study suggests that Fru-AGE is present in appreciable levels in common beverages and condiments (Figure 9), and exogenous, dietary Fru-AGE may contribute to the accumulation of Fru-AGE in the body. The increased usage of fructose in cooking may cause adverse health effects. AGEs form during the heating of common foods and, in contrast to in vivo AGEs formation, they can develop during cooking much more rapidly and in far greater concentrations. ${ }^{40}$ Approximately $10 \%$ of ingested AGEs (cooked with egg white and fructose) are absorbed, of which two-thirds are retained in tissues in reactive forms. ${ }^{40}$ Dietary AGEs have been shown to accelerate the progression of nephropathy and to shorten survival times in an animal model of diabetes. ${ }^{41}$ In human beings with diabetes, the mean concentration of C-reactive protein was $135 \%$ higher when the diet was high in AGEs than when cooking methods were altered to reduce the dietary AGEs content. ${ }^{42}$ Even if the lactic acid bacteria beverage of 25000 units is taken, concentration in the blood of Fru-AGE is $\sim 0.5 \mathrm{unit} / \mathrm{ml}(25000$ units $\times 0.1 / 5000 \mathrm{ml})$. Therefore, the amount of Fru-AGE in the blood suggests that it was formed in situ. However, an individual drinking Fru-AGE-rich beverages and foods every day may wish to restrict their intake. Additional clinical investigation may provide us with more information about whether restriction of food-derived AGEs (Fru-AGE and others) is beneficial for the prevention of the development of diabetes in high-risk patients and may be a novel therapeutic target to prevent diabetes and its related complications.

The contribution of fructose as an intracellular glycating agent may also be of importance. Fructose and fructose metabolites are located intracellularly, and in several organs, fructose is formed from glucose by the polyol pathway. The intracellular concentration of fructose also is elevated in those tissues of diabetic patients in which the polyol pathway is active, in particular the ocular lens, kidney, and peripheral nerves. ${ }^{6,736}$ As AR has a high $\mathrm{Km}$ for glucose, the polyol pathway is not very active at normal glucose levels. In hyperglycemia, however, glucose levels in insulinindependent tissues, such as brain and nerve tissue, kidney, lens, and the red blood cells, increase and, consequently, there is an increase in the activity of the polyol pathway. Fructose may be phosphorylated by hexokinase, an enzyme that is present in all cells; however, hexokinase has a strong preference for glucose, and glucose, which is present at about $5 \mathrm{mM}$ concentration in blood, is a strong competitive inhibitor of the phosphorylation of fructose. The newly synthesized fructose can be transported or leak 


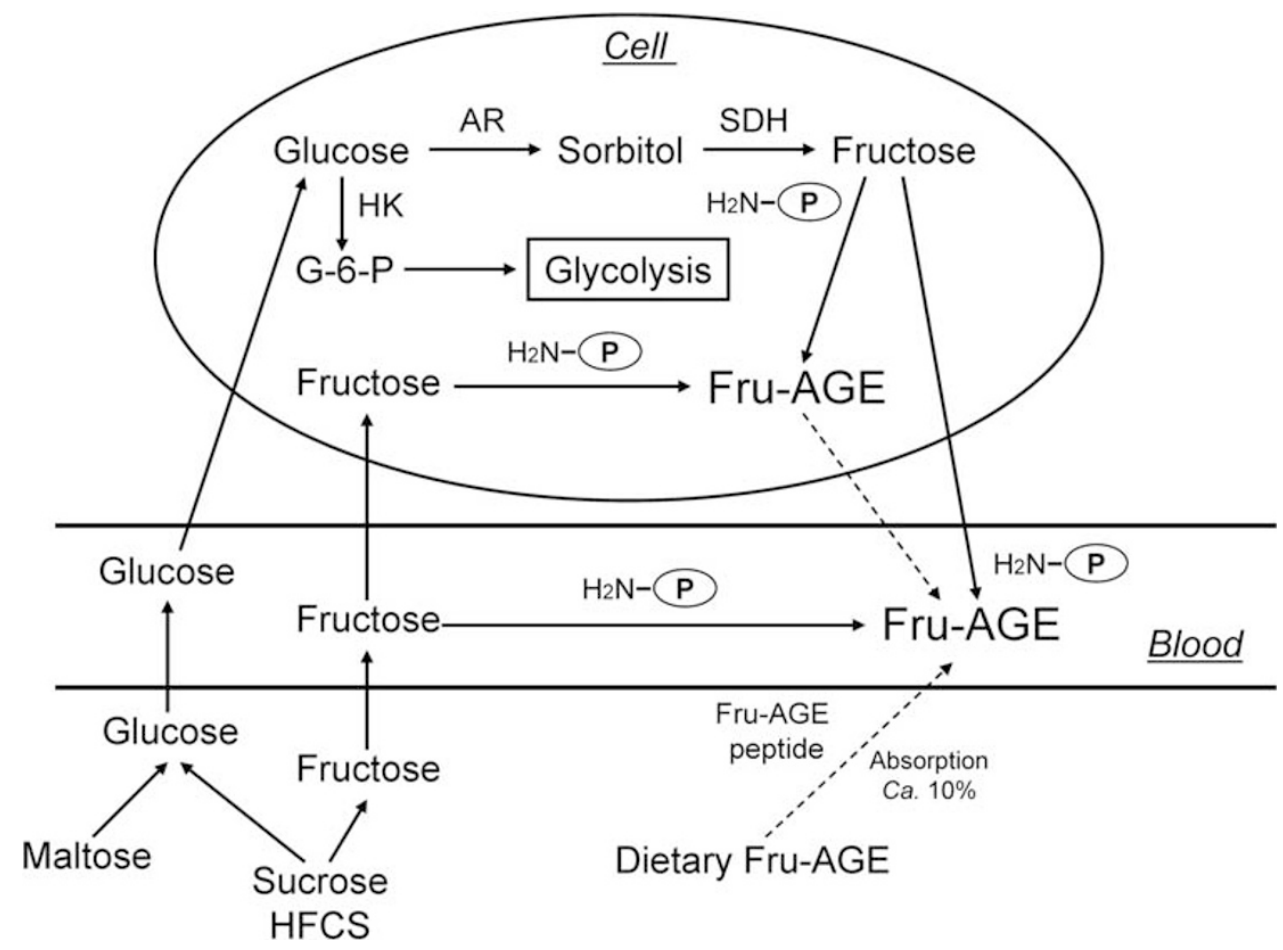

Figure 10 Alternative routes for the formation of Fru-AGE in vivo. Under hyperglycemic conditions, an increased intracellular glucose concentration stimulates the polyol pathway to generate fructose in insulin-independent tissues such as brain and nerve tissue, kidney, lens, and red blood cells. ${ }^{6,7,36}$ The newly synthesized fructose can be transported or leak passively across the plasma membrane. Fructose promotes the formation of Fru-AGE both intracellularly and extracellularly. Further, fructose, a component of table sugar (sucrose) and high-fructose corn syrup (HFCS), is also obtained from daily diets and beverages as well. ${ }^{31}$ Fructose can be thought to promote the advanced glycation end-products (AGEs) in blood and in the intracellular through the polyol pathway. $\mathrm{HK}$, hexokinase; $\mathrm{AR}$, aldose reductase; $\mathrm{SDH}$, sorbitol dehydrogenase; $\mathrm{HFCS}$, high-fructose corn syrup; $\mathrm{P}-\mathrm{NH}_{2}$, free amino-residue of protein.

passively across the plasma membrane. Fructose promotes the formation of Fru-AGE both intracellularly and extracellularly.

It is possible that Fru-AGE formation by these endogenous and exogenous routes contributes importantly to the tissue pathology of diabetes and aging (Figure 10). There may be relevance to human biology, and this particular AGEs, as illustrated in this work, may be a biomarker of diabetic of high-fructose/Fru-AGE diet intake. As the search for relevant diabetes biomarkers is an important one, this study may provide a new index for study in diabetes.

\section{Limitations}

There are believed to be $>100$ AGE structures in vivo, some of which are structurally identified. ${ }^{35,43-49}$ In this study, we did not determine the epitope structure of Fru-AGE, although we found that the epitope recognized by the antibody used here was different from well-characterized AGE structures such as pentosidine, pyrraline, argpyrimidine, CML, CEL, or ENL (Figure $5 \mathrm{c}$ ), or other reducing sugar-derived and dicarbonyl-derived AGEs (Figure 5a and b). Therefore, we do not know at present whether the structure of Fru-AGE may be unique. Further, spectroscopic and biochemical analyses will be needed to clarify the issue.

\section{ACKNOWLEDGEMENT}

This work was supported in part by grants from Japan Society for the Promotion of Science (Grant-in-Aid for Scientific Research (B), \#19300254) and the Specific Research Fund of Hokuriku University.

\section{DISCLOSURE/CONFLICT OF INTEREST}

The authors declare no conflict of interest.

1. Barros $C M$, Lessa $R Q$, Grechi MP, et al. Substitution of drinking water by fructose solution induces hyperinsulinemia and hyperglycemia in hamsters. Clinics 2007:62:327-334.

2. Bray GA, Nielsen SJ, Popkin BM. Consumption of high-fructose corn syrup in beverages may play a role in the epidemic of obesity. Am J Clin Nutr 2004;79:537-543.

3. Heinig M, Johnson RJ. Role of uric acid in hypertension, renal disease, and metabolic syndrome. Cleve Clin J Med 2006;73:1059-1064.

4. Nakagawa T, Tuttle KR, Short RA, et al. Hypothesis: fructose-induced hyperuricemia as a causal mechanism for the epidemic of the metabolic syndrome. Nat Clin Pract Nephrol 2005;1:80-86.

5. Nakagawa $\mathrm{T}, \mathrm{Hu} \mathrm{H}$, Zharikov $\mathrm{S}$, et al. A causal role for uric acid in fructose-induced metabolic syndrome. Am J Physiol Renal Physiol 2006;290:F625-F631

6. Oates PJ. Polyol pathway and diabetic peripheral neuropathy. Int Rev Neurobiol 2002;50:325-392.

7. Maekawa K, Tanimoto T, Okada S. Gene expression of enzymes comprising the polyol pathway in various rat tissues determined by the comparative RT-PCR method. Jpn J Pharmacol 2002;88:123-126.

8. Iwata T, Popescu NC, Zimonjic DB, et al. Structural organization of the human sorbitol dehydrogenase gene (SORD). Genomics 1995;26:55-62.

9. Gabbay KH, Kinoshita JH. Mechanism of development and possible prevention of sugar cataracts. Isr J Med Sci 1972;8:1557-1561. 
10. Yaylayan VA, Huyghues-Despointes A. Chemistry of Amadori rearrangement products: analysis, synthesis, kinetics, reactions, and spectroscopic properties. Crit Rev Food Sci Nutr 1994;34:321-369.

11. Ortwerth BJ, Olesen PR. Ascorbic acid-induced crosslinking of lens proteins: evidence supporting a Maillard reaction. Biochim Biophys Acta 1988;956:10-22.

12. Bunn HF, Higgins PJ. Reaction of monosaccharides with proteins: possible evolutionary significance. Science 1981;213:222-224.

13. Giardino I, Edelstein D, Brownlee M. BCL-2 expression or antioxidants prevent hyperglycemia-induced formation of intracellular advanced glycation endproducts in bovine endothelial cells. J Clin Invest 1996:97:1422-1428.

14. Giardino I, Edelstein D, Brownlee M. Nonenzymatic glycosylation in vitro and in bovine endothelial cells alters basic fibroblast growth factor activity. A model for intracellular glycosylation in diabetes. J Clin Invest 1994;94:10-17.

15. Suarez G, Rajaram R, Oronsky AL, et al. Nonenzymatic glycation of bovine serum albumin by fructose (fructation). Comparison with the Maillard reaction initiated by glucose. J Biol Chem 1989;264: 3674-3679.

16. Dills Jr WL. Protein fructosylation: fructose and the Maillard reaction. Am J Clin Nutr 1993;58(5 Suppl):779S-787S.

17. McPherson JD, Shilton $\mathrm{BH}$, Walton DJ. Role of fructose in glycation and cross-linking of proteins. Biochemistry 1988;27:1901-1907.

18. Takeuchi M, Makita Z, Yanagisawa K, et al. Detection of noncarboxymethyllysine and carboxymethyllysine advanced glycation end products (AGE) in serum of diabetic patients. Mol Med 1999;5:393-405.

19. Takeuchi M, Makita Z, Bucala R, et al. Immunological evidence that non-carboxymethyllysine advanced glycation end-products are produced from short chain sugars and dicarbonyl compounds in vivo. Mol Med 2000;6:114-125.

20. Takeuchi M, Bucala R, Suzuki T, et al. Neurotoxicity of advanced glycation end-products for cultured cortical neurons. J Neuropathol Exp Neurol 2000;59:1094-1105.

21. Takeuchi M, Yanase $\mathrm{Y}$, Matsuura N, et al. Immunological detection of a novel advanced glycation end-product. Mol Med 2001;7:783-791.

22. Takeuchi M, Watai T, Sasaki N, et al. Neurotoxicity of acetaldehydederived advanced glycation end products for cultured cortical neurons. J Neuropathol Exp Neurol 2003;62:486-496.

23. Lee FK, Cheung MC, Chung S. The human sorbitol dehydrogenase gene: CDNA cloning sequence determination and mapping by fluorescence in situ hybridization. Genomics 1994;21:354-358.

24. Guerrant G, Moss CW. Determination of monosaccharides as aldononitrile, $\mathrm{O}$-methyoxime, alditol, and cyclitol acetate derivatives by gas chromatography. Anal Chem 1984;56:633-638.

25. Fu M-X, Requena JR, Jenkins AJ, et al. The advanced glycation end product, Nepsilon-(carboxymethyl)lysine, is a product of both lipid peroxidation and glycoxidation reactions. J Biol Chem 1996;271: 9982-9986.

26. Yabe-Nishumura C. Aldose reductase in glucose toxicity: a potential target for the prevention of diabetic complications. Pharmacol Rev 1998;50:21-33.

27. Matsuoka T, Kajimoto $\mathrm{Y}$, Watada $\mathrm{H}$, et al. Glycation-dependent, reactive oxygen species-mediated suppression of the insulin gene promote activity in HIT cells. J Clin Invest 1997;99:144-150.

28. Unoki $\mathrm{H}$, Yamagishi S. Advanced glycation end products and insulin resistance. Curr Pharm Design 2008;14:987-989.
29. Yamagishi S, Ueda S, Okuda S. Food-derived advanced glycation end products (AGEs): a novel therapeutic target for various disorders. Curr Pharm Design 2007;13:2832-2836.

30. Vlassara $\mathrm{H}$. Advanced glycation in health and disease: role of the modern environment. Ann NY Acad Sci 2005;1043:452-460.

31. Gaby AR. Adverse effects of dietary fructose. Altern Med Rev 2005;10:294-306.

32. Bucala R, Cerami A. Advanced glycosylation: chemistry, biology, and implications for diabetes and aging. Adv Pharmacol 1992;23:1-34.

33. Thornalley PJ. Pharmacology of methylglyoxal: formation, modification of proteins and nucleic acids, and enzymatic detoxification. A role in pathogenesis and antiproliferative chemotherapy. Gen Pharmacol 1996;27:565-573.

34. Thornalley PJ, Langborg A, Minhas HS. Formation of glyoxal, methylglyoxal and 3-deoxyglucosone in the glycation of proteins by glucose. Biochem J 1999;344:109-116.

35. Takeuchi M, Makita Z. Alternative routes for the formation of immunochemically distinct advanced glycation end-products in vivo. Curr Mol Med 2001;1:305-315.

36. Schalkwijk CG, Stehouwer CD, van Hinsbergh VW. Fructose-mediated non-enzymatic glycation: sweet coupling or bad modification. Diabetes Metab Res Rev 2004;20:369-382.

37. Riby JE, Fujisawa T, Kretchmer N. Fructose absorption. Am J Clin Nutr 1993:58(5 Suppl):748S-753S.

38. Elliott SS, Keim NL, Stern JS, et al. Fructose, weight gain, and the insulin resistance syndrome. Am J Clin Nutr 2002;76:911-922.

39. Krajcovicova-Kudlackova M, Sebekova K, Schinzel R, et al. Advanced glycation end products and nutrition. Physiol Res 2002;51:313-316.

40. Koschinsky T, He CJ, Mitsuhashi T, et al. Orally absorbed reactive glycation products (glycotoxins): an environmental risk factor in diabetic nephropathy. Proc Natl Acad Sci USA 1997;94:6474-6479.

41. Zheng F, He C, Cai W, et al. Prevention of diabetic nephropathy in mice by a diet low in glycoxidation products. Diabetes Metab Res Rev 2002;18:224-237.

42. Vlassara H, Cai W, Crandall J, et al. Inflammatory mediators are induced by dietary glycotoxins, a major risk factor for diabetic angiopathy. Proc Natl Acad Sci USA 2002;99:15596-15601.

43. al-Abed Y, Kapurniotu A, Bucala R. Advanced glycation end products: detection and reversal. Methods Enzymol 1999;309:152-172.

44. Baynes JW, Thorpe SR. Role of oxidative stress in diabetic complications. A new perspective on an old paradigm. Diabetes 1999;48:1-9.

45. Nagai $R$, Hayashi $C M$, Xia $L$, et al. Identification in human atherosclerotic lesions of GA-pyridine, a novel structure derived from glycolaldehyde-modified proteins. J Biol Chem 2002;277:48905-48912.

46. Tessier FJ, Monnier VM, Sayre LM, et al. Triosidines: novel Maillard reaction products and cross-links from the reaction of triose sugars with lysine and arginine residues. Biochem J 2003;369:705-719.

47. Monnier VM. Intervention against the Maillard reaction in vivo. Arch Biochem Biophys 2003;419:1-15.

48. Usui T, Hayase F. Isolation and identification of the 3-hydroxy-5hydroxymethyl-pyridinium compound as a novel advanced glycation end product on glyceraldehyde-related Maillard reaction. Biosci Biotechnol Biochem 2003;67:930-932.

49. Ahmed N, Battah S, Karachalias N, et al. Increased formation of methylglyoxal and protein glycation, oxidation and nitrosation in triosephosphate isomerase deficiency. Biochim Biophys Acta 2003;1639:121-132. 